\title{
ESTILOS DE APRENDIZAGEM E NÍVEIS DE COMPROMETIMENTO ORGANIZACIONAL: UMA ABORDAGEM COM RESIDENTES MULTIPROFISSIONAIS DA SAÚDE ${ }^{1}$

\author{
LEARNING STYLES AND ORGANIZATIONAL \\ COMMITMENT LEVELS: AN APPROACH \\ TO HEALTH MULTIDISCIPLINARY
}

Recebido 06/08/2012

Aceito $01 / 12 / 2012$

Katiuscia Schiemer Vargas ${ }^{2}$ Jucelaine Arend Birrer ${ }^{3}$ Italo Fernando Minello ${ }^{4}$

\section{RESUMO}

O artigo investiga a relação entre os estilos de aprendizagem (E.A.) e os níveis de comprometimento organizacional (C.O.) de multiprofissionais da saúde para com um Programa de Residência Multiprofissional em Saúde de uma Instituição Federal de Ensino Superior. Caracteriza-se como um estudo de caso de natureza quantitativa do tipo descritivo. Para a coleta dos dados utilizou-se o Inventário de E.A. de Kolb (1976) e a Escala de C.O. de Meyer, Allen e Smith (1993), validada no Brasil por Medeiros e Enders (1998). Como resultados têm-se: (i) quanto ao E.A., predominou o E.A. divergente, todavia, foram encontrados entre os pesquisados todos os E.A. definidos por Kolb (1976); (ii) com relação ao C.O., há comprometimento predominantemente de maneira afetiva com o Programa de Residência e, de forma oposta, há um comprometimento menor no nível instrumental e afiliativo; quanto à relação entre o E.A. e nível de C.O., há uma relação entre os E.A. assimilador e divergente com os níveis 3 e 4 de C.O., contudo, os E.A. acomodador e convergente não possuem relação com os níveis de C.O.

Palavras-chave: Aprendizagem, Comprometimento, Vínculo, Residência.

\footnotetext{
${ }^{1}$ Este manuscrito não teve fonte de financiamento de pesquisa.

2 Mestranda em Administração na Universidade Federal de Santa Maria (UFSM), Santa Maria, Rio Grande do Sul, Brasil. E-mail: kati_schiemer@yahoo.com.br

${ }^{3}$ Mestranda em Administração na Universidade Federal de Santa Maria (UFSM), Santa Maria, Rio Grande do Sul, Brasil. E-mail: juarend@ibest.com.br

${ }^{4}$ Professor do Departamento de Ciências Administrativas da Universidade Federal de Santa Maria (UFSM), Santa Maria, Rio Grande do Sul, Brasil. E-mail: italo.minello@uol.com.br
} 


\section{ABSTRACT}

The paper discusses the relationship between learning styles (EA) and the levels of the organizational commitment (OC) of the multidisciplinary health for a Multidisciplinary Residency Program in Health from a Federal Institution of Higher Education. It is characterized as a case study quantitative descriptive. To collect data we used the Inventory of Kolb EA (1976) and the Scale of CO Meyer, Allen and Smith (1993), validated in Brazil by Medeiros and Enders (1998). As a result, we have: (i) regarding the EA, the EA divergent prevailed, however, were found among the all respondents EA defined by Kolb (1976), (ii) with respect to CO, there is so predominantly affective commitment to the Residency Program and, opposite, there is a minor compromise in the level instrumental and affiliated; regarding the relationship between EA and CO level, there is a correlation between EA and diverging assimilator with levels 3 and 4, CO, however the EA accommodative and convergent bear no relation to the CO levels.

Keywords: Learning. Commitment. Interaction. Residency.

\section{INTRODUÇÃO}

As questões relativas ao mundo globalizado, como a acirrada competitividade, necessidade de ser pró-ativo, diferenciação e criação de vantagem competitiva frente à concorrência conduzem as organizações na busca por colaboradores engajados e comprometidos com sua missão, visão e objetivos organizacionais a fim de atenderem os desafios que lhes são impostos pelas rápidas transformações da sociedade pós-industrial. (BASTOS, 2000).

Os recursos humanos podem somar valor às organizações, para tanto, sugere-se um caminho viável por meio de uma gestão que seja comprometida com o desenvolvimento de seus talentos e considere a mudança e as inovações internas e externas, de tal modo que possam influenciar suas estratégias organizacionais (LAWLER III, 2005). Nesse sentido, o comprometimento no trabalho reúne ações, atitudes e pensamentos manifestados pelo indivíduo em busca de realização pessoal e profissional (BATISTA E GONÇALVES, 2011).

Para Bandeira et al. (2000), o comprometimento pode ser entendido como um forte vínculo do indivíduo com a organização, que o estimula a dar sua energia e lealdade. Cada indivíduo pode vir a expressar diferentes níveis de comprometimento, isto se deve a características de seu comportamento, da satisfação com o que realizam, de seu bem-estar, dos benefícios oferecidos, da internalização dos valores e dos objetivos organizacionais propostos; ou até mesmo do envolvimento afetivo e das oportunidades que a empresa possa vir a oferecer (BANDEIRA et al., 2000; DAVIES et al., 1997; BASTOS e MAIA, 2011).

Na concepção de Probst e Büchel (1997), Gonçalo e Borges (2010) o indivíduo vive dentro da organização processos nos quais seus conhecimentos e sua base de valores podem vir a se modificar, podendo ser um indicador de desenvolvimento de habilidades para resolver problemas organizacionais, de sua capacidade de decidir e agir, processo este denominado de aprendizagem organizacional. Para os autores, este processo leva ao desenvolvimento de capacidades e características oriundas da interação entre diferentes membros dentro de uma organização e suas relações, tendo por base o aprendizado organizacional e o aprendizado individual, sendo o último, caracterizado de forma particular em cada indivíduo devido às diferenças de valores, crenças, experiências de vida, estilos cognitivos e capacidade de adaptação às mudanças.

Dessa forma, pressupõe-se que as organizações, em especial as da saúde estão frente a um novo desafio, sendo necessário à definição de uma posição estratégica em relação à aquisição e transferência do conhecimento (GONÇALO E BORGES, 2010; ANTONELLO e GODOY, 2011). Várias estratégias têm sido implantadas pelas organizações que prestam serviços de saúde com a finalidade de atender às exigências decorrentes do processo de globalização na perspectiva de 
gerar respostas rápidas e eficientes, que demandem, entre outros fatores, condições favoráveis ao indivíduo para aprender com os adventos do meio (CECCIN; FERLA, 2009).

Diante desse cenário, e considerando que o processo de aprendizagem é dirigido pelas necessidades e objetivos individuais, os estilos de aprendizagem se tornam altamente individuais tanto na direção como no processo (KOLB, 1997). Acompanhando o raciocínio do autor, os estilos de aprendizagem distintos são produzidos pela interação entre carreira, nível educacional e especialização de cada um, o que parece ser adequado considerar uma interação de habilidades analíticas e práticas de aprendizagem, pois engloba as visões opostas de experimentação ativa e de observação reflexiva.

Neste sentido, o conceito de estilo de aprendizagem pode ser representado, simultaneamente, por três componentes: o modo que se processa as informações; a relação dinâmica de estratégias de aprendizagem; e a percepção do indivíduo em relação a sua aprendizagem (CARDOSO e JANDL, 2012).

A partir do exposto e considerando a relevância da temática, este artigo tem como proposta norteadora mapear os estilos de aprendizagem e o nível de comprometimento de um grupo de multiprofissionais da saúde de um programa de residência multiprofissional em saúde de uma instituição de ensino superior na região central do estado do Rio Grande do Sul. O objetivo deste artigo consiste em investigar a relação entre os estilos de aprendizagem e os níveis de comprometimento dos multiprofissionais da saúde.

Para fins deste estudo, adotou-se o conceito de estilos de aprendizagem proposto por Kolb (1976), denominado como vivencial que considera como a experiência se traduz em conceitos. No que se refere ao comprometimento, adotou-se o conceito de Bastos (1994) como sendo um conjunto de sentimentos/ações que o indivíduo desenvolve pela organização em que trabalha.

$\mathrm{O}$ artigo está estruturado em seis seções. A primeira corresponde à introdução, a segunda e a terceira fornece o referencial teórico que abarca os conceitos de estilos de aprendizagem e de comprometimento organizacional. A quarta seção apresenta o método; a quinta apresenta os resultados e análise dos dados. A sexta e última seção se constitui das considerações finais do estudo.

\section{APRENDIZAGEM}

As decisões tomadas no cotidiano da vida do ser humano exigem deste um pensamento crítico agregado a estratégias que deem conta do processo de conhecimento. Essa situação depende muito da forma como diferentes habilidades de adaptação são desenvolvidas ao longo da vida pessoal e profissional (TERRA, 2001; FRANCO e MERHY, 2012), assim como, a representatividade que o indivíduo atribui a estes eventos; o que para Diniz (2007), representa diferentes preferências de perceber, reter, processar e organizar o conhecimento.

Visto sob a perspectiva de um ambiente de aprendizagem, essa heterogeneidade de preferências ocorre em consequência da existência de diferentes tipos de aprendizes (REIS et al., 2012). O processo de aprendizagem não é vivenciado por todos os indivíduos da mesma maneira, uma vez que o estilo de aprendizado que cada um possui é resultado de sua bagagem hereditária, experiências pessoais e exigências do ambiente em que vive, acarretando na sobreposição de algumas habilidades em relação à outras (KOLB, 1984; DINIZ, 2007; GRANITO, 2008; SANTOS, 2009).

Nesse sentido, Kuri (2004) enfatiza a importância de conhecer e compreender as diferenças culturais e a maneira como cada pessoa aprende, pois estimula o aprendizado de maneira mais eficaz; visto que, o conhecimento e a análise dos estilos de aprendizagem oferecem indicadores que auxiliam na interação entre as características de cada indivíduo e as realidades 
existenciais vivenciadas, gerando assim o autoconhecimento.

Muitos modelos foram criados e propostos por pesquisadores para medir as dimensões dos estilos de aprendizagem e suas tipologias, no intuito de explicar como se dão os processos que geram o aprendizado, a Escala de Estilos de Aprendizagem do Estudante (GRASHA, 1972); o Inventário de Estilos de Aprendizagem (KOLB, 1976); os Testes das Figuras Encaixadas (WITKIN et al., 1977); o Inventário de Estilo de Interesse Cognitivo (NUNNEY, 1978); o Índice de Estilos de Aprendizagem (FELDER E SOLOMAN, 1991); e o Inventário Brasileiro para Diagnóstico das Diferenças Individuais (CASADO, 1993), que tem sua teoria relacionada a tipos psicológicos.

Para fins deste estudo, se adotou o Inventário de Estilos de Aprendizagem de Kolb (KOLB, 1976), o qual contempla uma abordagem descritiva de como o indivíduo gera, a partir de sua experiência, os conceitos que guiarão seu comportamento em situações de aprendizagem e como ele modifica esses conceitos a fim de aumentar sua eficiência.

Este modelo pressupõe que o processo de aprendizagem é composto por quatro etapas consecutivas: experiência concreta (EC); observação reflexiva (OR); conceituação abstrata (CA); e, experimentação ativa (EA). De modo mais palpável (Figura 1), a ideia do processo é se envolver completa, aberta e imparcialmente em novas experiências (EC), realizar reflexões e observações sobre essas experiências e sobre seu contato com o mundo (OR), criar conceitos abstratos e generalizações que integrem suas observações em teorias sólidas em termos de lógica (CA) e; usar essas teorias para tomar decisões e resolver problemas e testar os resultados e suas implicações em novas situações por meio de experimentação ativa (EA), retornando-o ao início do ciclo para novas vivências concretas e assim sucessivamente (CRISI e BRITTO, 2004).

O modelo de Kolb (KOLB, 1997) trabalha como um instrumento para identificação individual do estilo de aprendizagem - Inventário de Estilos de Aprendizagem, o qual é aplicado neste estudo. A partir da classificação atribuída para cada sentença, o inventário mede a ênfase de cada indivíduo para cada uma das quatro habilidades que considera no processo de aprendizagem (EC, $\mathrm{OR}, \mathrm{CA}$ e EA) e por fim indica o grau em que o indivíduo prioriza a abstração sobre a concretude (CA-EC) e a experimentação ativa sobre a reflexão (EA-OR).

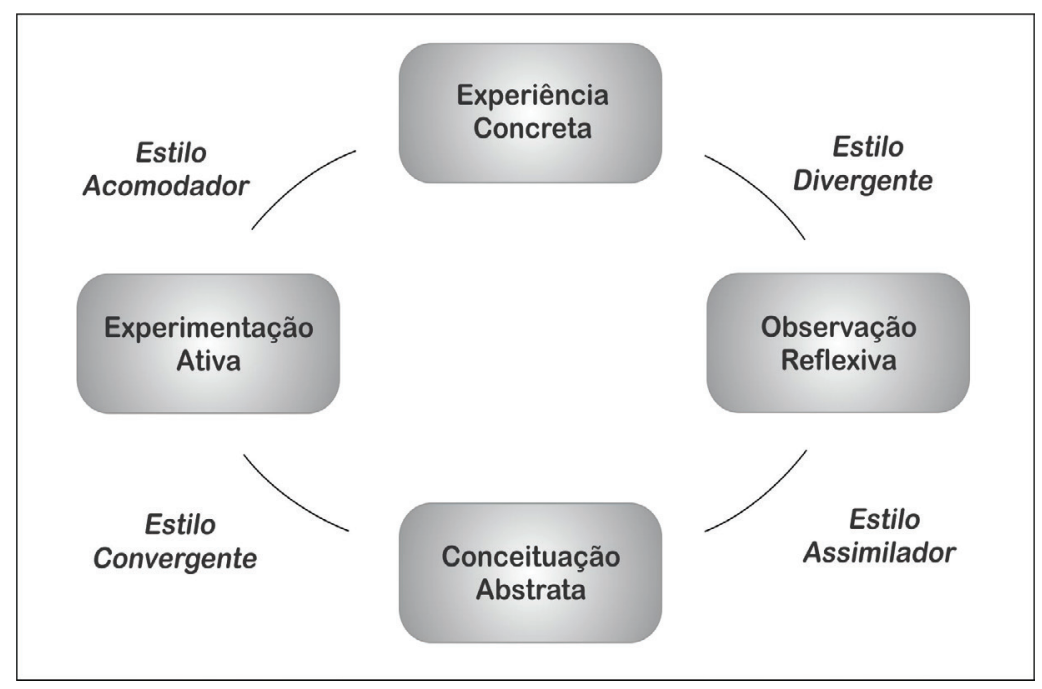

Figura 1: Inventário de Estilos de Aprendizagem de Kolb (1976). Fonte: Baseado em Kolb (1976).

A partir do reconhecimento das habilidades de aprendizado de cada indivíduo, o modelo identifica quatro estilos de aprendizagem que caracterizam a maneira de aprender de cada um, cada 
um com suas características (KOLB, 1976), são eles: acomodador; convergente; assimilador; e, divergente. A Tabela 1 apresenta as características e os estilos de aprendizagem propostos pelo autor.

Tabela 1: Características e Estilos de Aprendizagem de Kolb (1976).

\begin{tabular}{|c|c|c|}
\hline $\begin{array}{c}\text { CARACTERÍSTICA DE } \\
\text { APRENDIZAGEM }\end{array}$ & DESCRIÇÃO & $\begin{array}{c}\text { ESTILO DE APRENDI- } \\
\text { ZAGEM }\end{array}$ \\
\hline $\begin{array}{l}\text { Experiência Concreta } \\
\text { (EC) }\end{array}$ & $\begin{array}{l}\text { Fase do aprendizado na qual os indivíduos tendem } \\
\text { a preferir vivenciar situações de forma concreta, en- } \\
\text { volvendo-se em circunstâncias reais. A característica } \\
\text { nesta fase é de instigar novas ideias e perspectivas. }\end{array}$ & Divergente \\
\hline $\begin{array}{l}\text { Observação Reflexiva } \\
\text { (OR) }\end{array}$ & $\begin{array}{l}\text { Fase em que se prefere observar e refletir cautelo- } \\
\text { samente sobre a realidade antes de tomar posições. } \\
\text { Nesta fase se valoriza a organização das ideias, sua } \\
\text { classificação e ordenamento por escalas lógicas de } \\
\text { valor. Usualmente se apresenta predisposição para } \\
\text { reter e trabalhar grande número de informações. }\end{array}$ & Assimilador \\
\hline $\begin{array}{l}\text { Conceituação Abstrata } \\
\text { (CA) }\end{array}$ & $\begin{array}{l}\text { Fase em que conceitos e ideias causam impacto } \\
\text { profundo, antes mesmo da realidade, possibilitan- } \\
\text { do "experiências" mentais que preparam para situ- } \\
\text { ações reais. Transitando inicialmente dentro desse } \\
\text { mundo ideal, as pessoas nessa fase, possuem incli- } \\
\text { nação para direcionar suas reflexões em busca de } \\
\text { uma resposta compreensível, são voltadas a propor } \\
\text { soluções rápidas e testá-las para certificar-se de suas } \\
\text { conclusões. }\end{array}$ & Convergente \\
\hline $\begin{array}{l}\text { Experimentação Ativa } \\
\text { (EA) }\end{array}$ & $\begin{array}{l}\text { Fase onde se está munido dos conceitos de que já } \\
\text { se dispõe de antemão. A preferência dos membros } \\
\text { nesta fase conduz à visão de como as coisas funcio- } \\
\text { nam, uma vez que têm habilidade para enfrentar a } \\
\text { realidade à medida que se deparam com ela, sem } \\
\text { preparações anteriores, enfrentam situações novas } \\
\text { com grande versatilidade. }\end{array}$ & Acomodador \\
\hline
\end{tabular}

Fonte: Adaptado de Kolb (1976).

Diante do exposto, cada indivíduo confere maior destaque à etapa em que melhor se adapta e assim é capaz de absorver mais informações nesta do que nas demais. Operando dentro da etapa em que mais se identifica, o indivíduo poderá produzir aprendizado com melhores resultados (CRISI e BRITO, 2004). Nesse sentido, a maneira como os indivíduos aprendem nas organizações, possui grande relevância como subsídio para investimentos na área de gestão de pessoas (BIDO, et al., 2010) na potencialização dos fatores favoráveis ao comprometimento nas organizações.

\section{COMPROMETIMENTO ORGANIZACIONAL}

O termo comprometimento é comumente utilizado quando alguém se refere a relações que se estabelecem entre pessoas, entre uma pessoa e um grupo ou uma pessoa e uma organização (BASTOS e MAIA, 2011). Podendo também ser entendido como uma expressão de vínculo do indivíduo com a organização, despertando uma necessidade de interação como sinônimo de lealdade do indivíduo (BANDEIRA et al., 2000; MEDEIROS et al.,1998).

De maneira mais especifica, o comprometimento pode ser percebido por três linhas de abordagem: uma aproximação de compromisso e envolvimento; um sinônimo de lealdade individual; e, um prejuízo, o qual pode refletir de forma negativa na vida do indivíduo (BASTOS, 1994b).

Neste sentido, o comportamento organizacional tem sido estudado sob diferentes en- 
foques, sendo predominantes o enfoque afetivo, o enfoque instrumental/calculativo e o enfoque normativo, os quais são tratados de forma isolada, ou em modelos multidimensionais (MEDEIROS, 2003). Essas proposições passaram a ser consideradas após a percepção de alguns pesquisadores de que os enfoques unidimensionais mais do que tipos de comprometimento eram componentes presentes no vínculo psicológico entre indivíduo e organização. Alguns autores, com a finalidade de descrever a presença de mais de um tipo de enfoque, utilizam expressões como: tipologia do comprometimento (MOWDAY, 1982); dimensões do comprometimento (BECKER, 1992); e, componentes do comprometimento (MEYER e ALLEN, 1991).

Em se tratando da expressão, Para Meyer e Allen (1991) os "componentes do comprometimento" podem ser divididos em três bases: a afetiva (affectivo), permanência do indivíduo na organização por livre e espontânea vontade; a instrumental (continuance), engajamento do indivíduo com a organização, está ligado a sua permanência por necessidade, também denominado de side bet, assumindo outras denominações como calculativo e/ou continuação (BASTOS, 1994b); e, finalmente, a normativa (obligation), se refere a um sentimento de obrigação em permanecer na organização uma vez que está associado a valores e deveres morais. O pensamento de desligamento gera uma culpa ao indivíduo como se estivesse cometendo uma traição.

No caso do Brasil foram realizados dois estudos visando à validação do modelo de Meyer e Allen (1991). No primeiro (MEDEIROS e ENDERS, 1998), os índices de precisão obtidos para a realidade brasileira foram de 0,68 para a medida de comprometimento afetivo, 0,70 para normativo e 0,61 para o instrumental, os quais denotam uma fraca consistência interna (PESTANA e GAGEIRO, 2003). No segundo (MEDEIROS, 2003), repetiu-se a análise do modelo de três componentes do comprometimento organizacional de Mayer e Allen (1991), encontrando-se um quarto componente, denominado de afiliativo (MEDEIROS, 2003).

O comprometimento normativo pode ser considerado, segundo Wierner (1982), como a soma de todas as forças normativas internalizadas, com a finalidade de preservar e alcançar os propósitos e objetivos organizacionais. Um estudo exploratório dos múltiplos componentes do comprometimento organizacional (MEDEIROS et al., 2005) corrobora esse conceito, propondo por em ação a aceitação de valores e objetivos organizacionais, os quais tem significado de controle sobre as ações das pessoas, sendo assim denominado de normativo-instrumental.

Diante disso, optou-se utilizar o modelo de conceitualização de três componentes do comprometimento organizacional proposto por Medeiros e Enders (1998), relacionando-o aos estilos de aprendizagem de Kolb (1976).

\section{METODOLOGIA}

O presente estudo caracteriza-se como quantitativa do tipo exploratório (SAMPIERI et al., 2006; HAIR et al., 2005; RICHARDSON, 2011) baseado em um estudo de caso (GODOY, 1995; AMARATUNGA et al., 2002; YIN, 2010). Exploratório por se tratar de um estudo dentro de um contexto específico (programa de residência multiprofissional), o qual ainda está pouco explorado e apresenta uma parca literatura sobre a relação da aprendizagem com o comprometimento.

A coleta de dados foi feito a partir da aplicação de dois instrumentos: o Inventário de Estilos de Aprendizagem de Kolb (1976); e, a Escala de Comprometimento Organizacional de Meyer, Allen e Smith (MEYER et al., 1993; MEDEIROS et al., 1998).

O instrumento de Kolb (1976) é composto de sentenças às quais deve ser atribuída uma classificação de 1 a 4 , sendo o número 4 a representação da expressão que o participante melhor julga caracterizar a sua maneira de aprender e, o número 1 , aquela que pior caracteriza seu estilo 
de aprendizagem. A Escala de Comprometimento Organizacional de Meyer, Allen e Smith (MEYER et al., 1993; MEDEIROS et al., 1998) é composta por uma escala Likert de 7 (sete) pontos, sendo 1, "Discordo Totalmente" e 7 "Concordo Totalmente".

Como população-alvo definiu-se uma amostra de 30 multiprofissionais da saúde escolhidos aleatoriamente conforme disponibilidade para responder a pesquisa. Esta seleção caracterizou-se pela escolha intencional ou seleção racional (BARROS e LEHFELD, 2007) denominada de "purposeful sampling" (PATTON, 1990) em que o critério de inclusão é definido em relação a atributos do grupo de interesse. Os pesquisados pertencem a uma linha de cuidado ${ }^{2}$ dentre as quatro linhas que fazem parte de um programa de residência multiprofissional com "ênfase hospitalar" de uma instituição federal de ensino superior, localizada na região central do estado do Rio Grande do Sul.

A referida coleta foi realizada em 3 (três) etapas. A primeira corresponde à obtenção de dados relativos ao perfil dos respondentes; a segunda contém o instrumento de estilos de aprendizagem; e a terceira, a aplicação do instrumento de comprometimento organizacional.

Para a análise dos dados coletados, os mesmos foram tabulados, fazendo uso do software Microsoft Office Excel ${ }^{\circledR}$ e analisados por meio de análise descritiva e por técnica exploratória de análise multivariada com auxílio do software "Statistica 6.0". Como análise descritiva utilizou-se a média (média geral e a média das variáveis do instrumento - afetivo, instrumental, normativo e afiliativo) e como técnica exploratória de análise estatística multivariada empregouse a Análise de Correspondência que permite a tabulação e análise cruzada de duas variáveis categóricas (HAIR et al., 2005).

A pesquisa foi submetida à avaliação do Comitê de Ética em Pesquisa (CEP), tendo como registro o número do CAAE: 01680712.6.0000.5346. Ao público pesquisado deu-se ciência de um Termo de Consentimento Livre e Esclarecido (TCLE), conforme preconiza a Resolução do Conselho Nacional de Saúde (CNS) № 196/96 (BRASIL, 2004). A coleta dos dados foi realizada no período de março a abril de 2012 pelos próprios pesquisadores no local de atuação dos multiprofissionais da saúde.

\section{APRESENTAÇÃO E DISCUSSÃO DOS RESULTADOS}

No intuito de facilitar a apresentação e compreensão da discussão dos resultados encontrados, dividiu-se este tópico em quatro partes: (a) perfil dos pesquisados; (b) estilos de aprendizagem; (c) níveis de comprometimento organizacional; e (d) o estudo da relação entre as variáveis.

\subsection{Perfil dos Pesquisados}

Dos 30 multiprofissionais da saúde pesquisados $94 \%$ pertencem ao gênero feminino e $6 \%$ ao masculino. Quanto à faixa etária, $86 \%$ possui idade entre 20 e 30 anos e $4 \%$ de 30 a 40 anos. Com relação à naturalidade, $37 \%$ são naturais da cidade onde o programa está vinculado, sendo que, $100 \%$ residem nesta mesma cidade.

No que se refere à profissão, $20 \%$ dos pesquisados são nutricionistas; $20 \%$ são enfermeiros; $14 \%$ são fisioterapeutas; as profissões de fonoaudiólogo, psicólogo, assistente social e farmacêutico correspondem cada uma, a 10\% e 3\% são cirurgiões dentistas e 3\% exercem a profissão de terapeuta ocupacional. Destes profissionais, 60\% graduaram-se entre os anos de 2010 e $2012,84 \%$ estão no seu 10 ano de atuação no programa de residência e $16 \%$ estão atuando no 20 ano.

${ }^{2}$ Segundo Merhy e Cecílio (2003), Linha de Cuidado (LC) está associada à imagem de uma linha de produção voltada ao fluxo de assistência ao usuário, centrada em seu campo de necessidades. 


\section{2 - Estilos de aprendizagem dos multiprofissionais da saúde}

Os primeiros estudos sobre o tema aprendizagem foi realizado pelos antigos hindus, há aproximadamente 2500 anos atrás, refletindo sobre como as pessoas aprendiam religião (CLAXTON; MURRELL, 1987). A evolução dos estudos sobre os estilos de aprendizagem, no início do século XX, passa pela identificação do estilo cognitivo. Em meados de 1900, através das pesquisas realizadas por psicólogos alemães, entre eles Jung, foram realizadas pesquisas envolvendo os tipos psicológicos. Foi a partir de 1921, por meio de estudiosos como Allport, Lowenfield, Klein, que se ampliou uma base conceitual sobre a qual se desenvolveu o estudo das diferenças entre os indivíduos e os estilos de aprendizagem (REIS et al., 2012).

Diante disso, os estilos de aprendizagem podem ser interpretados de diferentes formas considerando as características individuais, personalidade, a maneira como cada um processa a informação que chega ao seu conhecimento, o ambiente o qual está inserido, as pessoas as quais convive, entre outros componentes que fazem parte do cotidiano desse indivíduo.

Quanto aos estilos de aprendizagem dos multiprofissionais da saúde pesquisados, se identificaram os quatro estilos propostos por Kolb (1976), sendo que, o estilo predominante, foi o divergente, correspondendo a $67 \%$ dos pesquisados. Este estilo de aprendizagem parte da experiência concreta, ou seja, a prática profissional em lócus - o ambiente hospitalar - conduzindo-os para a observação reflexiva. Isso demonstra uma grande habilidade de imaginação, inovação e percepção dos acontecimentos sobre diversos ângulos, preferindo ouvir e partilhar novas ideias. Relaciona-se esse achado à proposta pedagógica do programa de residência, a qual possui como intenção formar profissionais para o Sistema Único de Saúde (SUS) contemplando as três estâncias do cuidado, ou seja, primária, secundária e terciária. (FEUERWERKER e MERHY, 2011), considerando estes espaços como troca de saberes.

A metodologia proposta pelo programa desafia estes profissionais a interagirem efetivamente por meio de vivências concretas em circunstâncias reais, fomentando novas formas de executar os processos de trabalho, no intuito de estimular a interação e a qualificação dos multiprofissionais em relação às necessidades do sistema de saúde pública. Esta proposta de formação pressupõe o desenvolvimento de competências para articular serviços, ações e políticas públicas de saúde, como forma de assegurar a construção de caminhos para a integralidade da assistência, com repercussão mais efetiva na saúde da população e na qualidade de vida dos indivíduos (UFSM, 2010).

O estilo assimilador, com $23 \%$ de representatividade entre os pesquisados define os multiprofissionais cujas características são a integração da experiência e do conhecimento, os quais buscam apresentar visão e organização lógica, são competentes em unir observações de experiências a conhecimentos anteriores, de modo a propor teorias, sendo a criação de modelos teóricos seu ponto forte. Essas pessoas se destacam quando se trata de entender uma ampla gama de informações, de modo a dar-lhe uma forma concisa e lógica.

Ao analisar os estilos divergente e assimilador em conjunto, verificou-se que estes representam o estilo de aprendizagem de $90 \%$ dos multiprofissionais da saúde pesquisados, o que pode servir de balizador para auxiliar na elaboração dos materiais didáticos do programa.

O estilo de aprendizagem acomodador correspondeu apenas a $7 \%$ e o estilo convergente a $3 \%$ entre multiprofissionais pesquisados.

\section{3 - Níveis de comprometimento organizacional}

Com base na análise descritiva empregada (média geral e a média das variáveis do instru- 
mento) constatou-se que o componente do comprometimento organizacional predominante entre os multiprofissionais da saúde pesquisados é o afetivo, seguido pelos componentes normativo, instrumental e afiliativo. A média geral do comprometimento apresentou-se no nível mediano (considerando a escala de 7 pontos utilizada), ou seja, nível 4 (quatro), conforme pode ser visualizado na Tabela 2.

Tabela 2: Médias Arredondadas dos Componentes do Comprometimento Organizacional.

\begin{tabular}{|l|c|l|}
\hline \multicolumn{1}{|c|}{$\begin{array}{c}\text { COMPONENTE DO COMPROMETIMENTO } \\
\text { ORGANIZACIONAL }\end{array}$} & MÉDIA & \multicolumn{1}{|c|}{ CLASSIFICAÇÃO } \\
\hline Afetivo & 5 & Concordo Parcialmente \\
\hline Instrumental & 3 & Discordo Parcialmente \\
\hline Normativo & 4 & Não concordo nem discordo \\
\hline Afiliativo & 3 & Discordo Parcialmente \\
\hline Comprometimento Geral & $\mathbf{4}$ & Não concordo nem discordo \\
\hline
\end{tabular}

Fonte: Pesquisa.

Com base na predominância do componente afetivo do comprometimento entre os multiprofissionais da saúde que participaram da pesquisa, entende-se que estes se identificam com o programa de residência. Isto pode ser um indicador de que estes indivíduos vivenciam experiências positivas e valores organizacionais de trabalho as quais podem estar relacionadas a um apego emocional.

O componente normativo do comprometimento ocupa o segundo nível predominante entre os pesquisados. Relaciona-se esse achado ao fato destes multiprofissionais serem recém- formados, terem participado de uma seleção pública a qual exigiu um esforço significativo para o seu ingresso nesta modalidade de formação, assim como o fato de poderem agregar a aquisição de conhecimento por meio da atuação prática com a remuneração da bolsa de estudo paga a estes multiprofissionais.

Percebe-se ainda, a partir dos resultados obtidos, que os multiprofissionais pesquisados estão comprometidos em menor escala nos componentes instrumental e afiliativo os quais apresentaram média de 3 (três).

Com relação ao Alfa de Cronbach das variáveis do constructo, obtiveram-se valores abaixo do considerado confiável. No entanto, obteve-se um valor de Alfa de Cronbach geral do constructo considerado confiável, já que o mesmo foi de 0,63 . Isso se sustenta na visão de que se deve considerar o valor mínimo de 0,6 para aceitação de confiabilidade da pesquisa dentro dos padrões estatísticos (HAIR et al., 2005).

5.4 - Relação entre os estilos de aprendizagem e os níveis de comprometimento organizacional

Com a finalidade de atingir o objetivo proposto para o presente estudo - investigar a relação entre os estilos de aprendizagem e os níveis de comprometimento dos multiprofissionais da saúde, a figura 2 demonstra a relação entre as variáveis obtida por meio da Análise de Correspondência. 
Figura 2: Relação entre Estilos de Aprendizagem e Níveis de Comprometimento Organizacional.

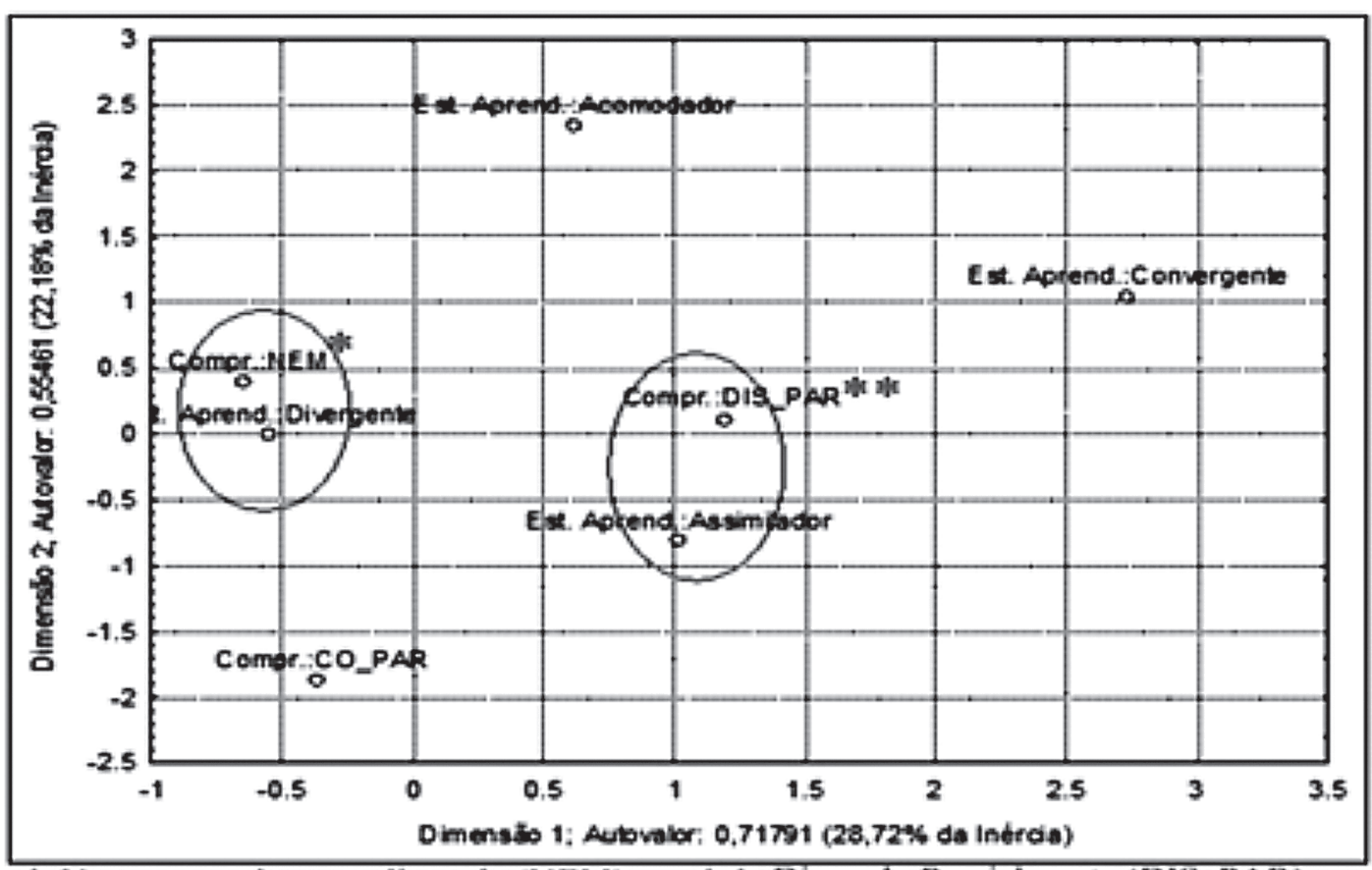

Nem concordo, nem discordo (NEM) 壮 Discordo Parcialmente (DIS_PAR)

Fonte: Pesquisa.

O estilo de aprendizagem divergente possui relação com o nível mediano de comprometimento, o qual corresponde na escala ao valor 4 (quatro). Esse resultado demonstra que os multiprofissionais pesquisados priorizam vivenciar as experiências e o aprendizado na prática e assim, realizam reflexões e observações sobre o que foi vivido. Dessa forma, não significa que estão mais ou menos comprometidos com o programa, pois seu foco é aproveitar ao máximo as oportunidades, podendo vir a ser uma fonte produtiva de aprendizagem para sua experiência profissional (SALOMÃO, 2011).

O estilo de aprendizagem assimilador possui relação com o nível 3 (três) da escala de comprometimento, sendo possível afirmar que se trata de indivíduos que não precisam mais da experimentação ativa do aprendizado, processam as informações de modo reflexivo e a partir disso criam "experiências mentais" (KOLB, 1976; SALOMÃO, 2011). Isto reflete o seu comprometimento com o programa de residência multiprofissional o qual fazem parte e, da profissão, podendo ser visualizado como um feedback de forma satisfatória.

Este resultado instiga a reflexão acerca da evolução das habilidades de aprender dos multiprofissionais da saúde pesquisados durante sua inserção no programa de residência, uma vez que, à medida que o indivíduo as modifica no sentido da experiência concreta em direção à conceituação abstrata, há uma diminuição do nível de comprometimento. Este pode ser um fator que pode restringir estes profissionais a aprender sobre diferentes enfoques. Essas observações podem também prover discussões internas no programa, no que diz respeito à abordagem teórico-prática, as metodologias de ensino e, sobretudo, nas atividades laborais vislumbrando a aplicabilidade destas de modo que os multiprofissionais permaneçam comprometidos.

Neste sentido, atribui-se as estratégias do referido programa a Constituição Federal de 1988 e a publicação da lei 8080/90 (BRASIL, 1990), que regulamentou o Sistema Único de Saúde (SUS), trazendo a tona discussões sobre a formação dos profissionais de saúde. A partir disso, foi possível a realização de mudanças nas práticas de saúde e alterações significativas no processo 
de formação e desenvolvimento de competências dos profissionais que estão sendo formados para o mercado de trabalho (CAVALHEIRO E GUIMARÃES, 2011).

Com base na realização deste estudo, a vivência do aprendizado parece estar associada a necessidades emergentes destes multiprofissionais em aplicar o conhecimento adquirido no período de graduação, buscando experiências concretas e aplicabilidade com o propósito de sanar possíveis lacunas deixadas por este período de formação. Associado a isso, por em prática a metodologia proposta pelo programa de residência. Porém, quando passam apenas a considerar a reflexão como modo de aprendizado, seu comprometimento tende a reduzir, o que pode estar relacionado à acomodação do profissional com o método tecnicista, uma das características vigentes dos serviços de saúde baseado no modelo biomédico (CECíLIO, 1997; FEUERWERKER e MERHY, 2011), assim como, pode estar relacionado a fatores gerenciais do programa.

No que se refere aos estilos de aprendizagem acomodador e convergente, estes não possuem relação com os níveis de comprometimento, o que faz emergir um ponto de reflexão para pesquisas futuras: perpassando pelas fases de conceituação abstrata, experimentação ativa e experiência concreta do processo de aprendizagem, o nível de comprometimento continuará a diminuir?

A partir disto, se percebe que modificando o estilo de aprendizagem os multiprofissionais pesquisados mudam também seu nível de comprometimento para com o programa de residência, i.e., à medida que o indivíduo altera suas habilidades de aprendizagem na direção experiência concreta - observação reflexiva - conceituação abstrata, ele diminui o seu nível de comprometimento na escala de 1 a 7, como se pode verificar na Figura 3.

Figura 3: Mudança de Estilo de Aprendizagem e Comprometimento Organizacional dos Multiprofissionais.

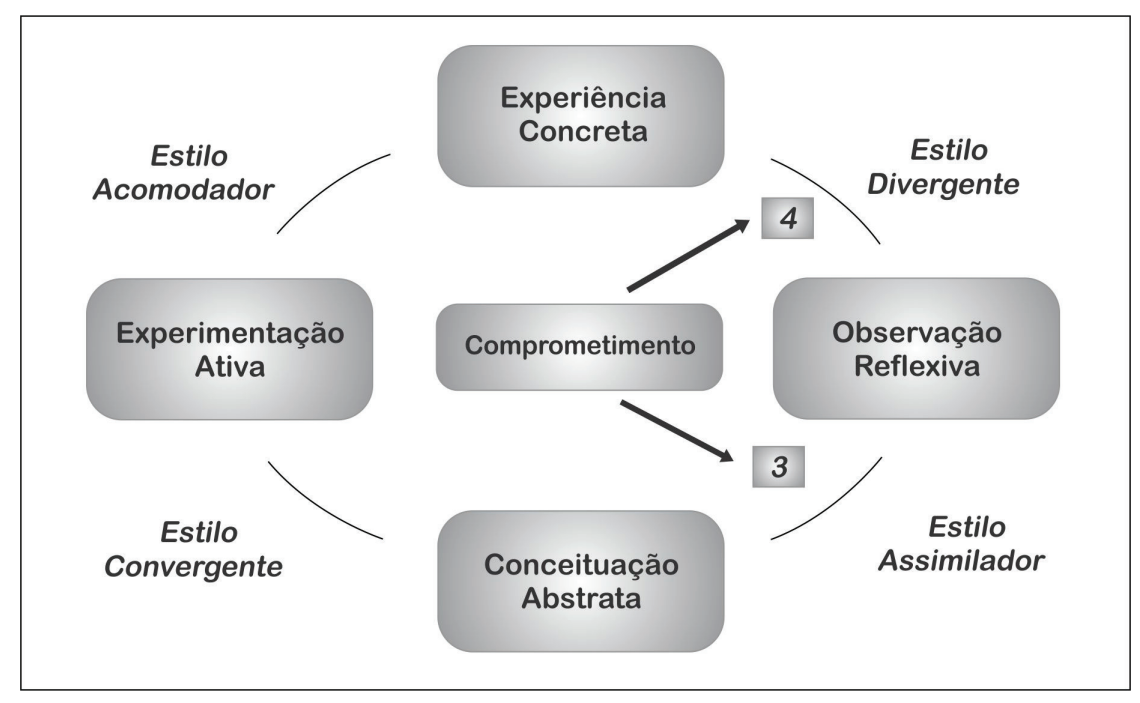

Fonte: Pesquisa.

Á medida que os multiprofissionais pesquisados transitam por uma fase do aprendizado vivenciam concretamente as experiências - Experiência Concreta; direcionando-se para a fase de observação e reflexão do que foi vivido - Observação Reflexiva; o que os caracteriza com o estilo de aprendizagem divergente, o qual está associado a um maior comprometimento. Partindo para a fase de Conceituação Abstrata do aprendizado, com o estilo de aprendizagem assimilador, os multiprofissionais apresentam um nível menor de comprometimento.

A análise referente a este resultado propõe discussões em relação as estratégias adotadas pelo programa, pois a medida que os indivíduos deixam de considerar a importância da vivência do 
aprendizado de maneira concreta e passam para uma fase do processo onde se verifica as informações, refletindo sobre as mesmas, eles parecem apresentar um menor nível de comprometimento.

Esse estudo pressupõe que os multiprofissionais de saúde pesquisados, podem estar apresentando um comportamento atípico, pois o momento de formação o qual estão vivenciando, se caracteriza como de aprendizado tanto prático como teórico, o que deveria ser um determinante para aprenderem por experiências concretas junto a outros indivíduos que fazem parte do programa e que possuem uma experiência mais ampliada. Outro ponto a ser considerado pode estar relacionado a fatores como sobrecarga de trabalho, carga horária prática e teórica elevada, aspectos organizacionais como: estrutura, organização das atividades, gerenciamento, centralização das decisões, feedback insuficiente, supervisão, acompanhamento inadequado, dificuldade de institucionalização do programa e demais elementos que possam estar agregados a gestão do programa.

Sendo assim, apesar dos multiprofissionais pesquisados estarem em uma fase do processo de aprendizagem que compreende o estilo divergente de aprender, ainda poderão passar por vivências e experiências no programa de residência que façam com que vivenciem outras etapas do processo e apresentem outros estilos de aprendizagem, assim como diferentes formas de se comprometerem.

\section{CONSIDERAÇÕES FINAIS}

A abordagem teórica deste artigo permitiu verificar que a temática norteadora vem sendo pesquisada sobre diferentes aspectos. No entanto, a associação entre as variáveis "estilos de aprendizagem" e "comprometimento organizacional" parece ainda ser incipiente, principalmente no que tange ao contexto da área da saúde. Neste artigo buscou-se a aplicabilidade empírica do tema com foco nesta área. Compondo a pesquisa teve-se uma amostra de um grupo heterogêneo de multiprofissionais da saúde, atuantes em uma linha de cuidado de um programa de residência multiprofissional em saúde.

Considerando o tema comprometimento, com base no modelo de Meyer, Allen e Smith (MEYER et al., 1993; MEDEIROS e ENDERS, 1998), o qual foi utilizado para os fins metodológicos desta pesquisa, se percebe que o público pesquisado se compromete predominantemente de maneira afetiva com o programa em estudo, o que indica um comprometimento com identificação do indivíduo com as metas metodológicas propostas, bem como a introjeção de seus valores, assumindo-os como próprios (MOWDAY et al., 1982). De forma oposta, percebe-se que os pesquisados comprometem-se menos no nível instrumental e afiliativo o que caracteriza um baixo comprometimento relacionado à maneira como ocorre à troca de saberes entre o indivíduo e o contexto vivenciado (BECKER, 1960). Essa característica pode estar relacionada ao fato de serem profissionais que tem sua atuação restrita ao período de dois anos, tempo de duração do curso da residência.

Quanto ao processo de aprendizagem, os multiprofissionais pesquisados demonstram aprender de maneira cíclica; onde fatores como vivências e experiências cotidianas, variáveis ambientais como carreira, trabalho, tarefas, competências, fazem com que estes indivíduos se encontrem em fases distintas do processo de aprender (KOLB, 1984; GRISI e BRITO, 2004; PAWLOWSKY, 2001; BIDO et al., 2011) levando-o a um comprometimento que também pode oscilar.

Na relação entre os estilos de aprendizagem e os níveis de comprometimento dos multiprofissionais pesquisados parece ser uma questão que mereça estudos mais profundos, pois o comprometimento por parte destes multiprofissionais acaba interferindo na sua maneira de aprender. Isto pode estar relacionado ao conceito de comprometimento, o qual se relaciona a aspectos psicossociais construídos ao longo do tempo e, por isso mesmo, mais estável e internaliza- 
do pelos indivíduos. Isto pode estar relacionado ao fato do tempo de formação desta modalidade de ensino ser apenas de dois anos, interferindo na dinâmica cognitiva destes indivíduos quanto ao nível de comprometimento.

Diante disso, entende-se que este modelo de formação, possa estar em construção/ desconstrução, haja vista que é um programa com implantação recente, em atuação no ano vigente a quarta turma. Essa particularidade parece contribuir para que o panorama esteja incipiente e em fase de ajustes.

Como limitações, considera-se o fato de que este estudo foi realizado somente com uma das linhas de cuidado do programa de residência, o que não permite a generalização dos resultados para as demais linhas e para os demais multiprofissionais do programa.

Outro ponto a ser considerado é a possibilidade de uma abordagem qualitativa, podendo vir a auxiliar na compreensão dos fenômenos em estudo e suas relações e interações, assim como na inclusão de novos construtos. 


\section{REFERÊNCIAS}

AMARATUNGA, D.; BALDRY, D.; SARSHAR, M.; NEWTON, R. Quantitative and Qualitative Research in the Built Environment: application of 'mixed' research approach. Work Study, v. 51, n. 1, p. 17-31, 2002.

ANTONELLO, C.S.; GODOY, A.S. Aprendizagem organizacional no Brasil. Porto Alegre: Bookman, 2011.

BANDEIRA, M. L.; MARQUES, A. L.; VEIGA, R. T. As Dimensões Múltiplas do Comprometimento Organizacional: um estudo na ECT/MG. Revista de Administração Contemporânea - RAC, v. 24, n. 2, p. 133-157, mai./ago. 2000.

BARROS, A. J. S.; LEHFELD, N. A. S. Fundamentos de Metodologia Científica. 3a Ed. São Paulo: Pearson Prentice Hall, 2007.

BASTOS, A. V. B. Comprometimento no Trabalho: a estrutura dos vínculos do trabalhador com a organização, a carreira e o sindicato. Tese (Doutorado em Psicologia), Universidade de Brasília. UnB, Brasília, 1994b.

BASTOS, A. V. B. Comprometimento do empregado e contextos organizacionais em mudança: o caso do Banco do Brasil. Revista de Ciências Humanas, Florianópolis, Edição Esp. Temática, p. 117-140, 2000.

BASTOS, A. V. B. O Conceito de Comprometimento: sua natureza e papel nas explicações do comportamento humano no trabalho. Organização \& Sociedade, v. 1, n. 2, p. 77-106, 1994.

BASTOS, A. V. B.; MAIA, L. G. Comprometimento calculativo e retaliação: visão integrada dos conceitos em uma organização pública. Rev. Adm. UFSM, Santa Maria, v. 4, n. 3, p. 390-405 set./dez. 2011.

BATISTA, K. B. C.; GONÇALVES, O. S. J. Formação dos Profissionais de Saúde para o SUS: significado e cuidado. Saúde Soc. São
Paulo, v.20, n.4, p.884-899, 2011.

BECKER, H. S. Notes on the Concept of Commitment. The American Journal of Sociology, v. 66, n.1, p. 32-40, 1960.

BECKER, T. E. Foci and Bases of Commitment: are they distinctions worth making? Academy of Management Journal, v. 35, n. 1, p. 232244, 1992.

BELNOSKI, A. M.; DZIEDZIC, M. O Ciclo de Aprendizagem na Prática de Sala de Aula. Athena - Revista Científica de Educação, v. 8, n. 8, jan./jun. 2007.

BIDO, D. S.; GODOY, A.S.; QUIROGA, G. M. M.; AMARAL, D.; YOSHIDA, E. C.; RIQUETTI, A. Relação entre a aprendizagem organizacional e o desempenho organizacional: uma abordagem baseada no conceito de estoques e fluxos de aprendizagem. XXXIV Encontro da ANPAD. Rio de Janeiro, 2010.

BIDO, D. S.; GODOY, A. S. FERREIRA, J. F.; KENSKI, J. M.; SCARTEZINI, V. N. Examinando a Relação entre Aprendizagem Individual, Grupal e Organizacional em uma Instituição Financeira. Revista Eletrônica de Administração, v. 17, n. 1, p. 58-85, jan/abr. 2011.

BRASIL. Lei no 8080, de 19 de setembro de 1990. Dispõe sobre as condições para a promoção, proteção e recuperação da saúde, a organização e o funcionamento dos serviços correspondentes e dá outras providências. Diário Oficial da União. Brasília, 20 set. 1990; Seção 1.

CARDOSO, S. M. V.; JANDL Jr., P. Estilos de Aprendizagem: aprendendo a aprender. Direito - UFS, v.5 n.2 p. 35-145, 1998.

CASADO, T. Tipos Psicológicos e Estilos de Comportamento Motivacional: o Diálogo entre Jung e Fromm. Dissertação (Mestrado em Administração), Faculdade de Economia, Administração e Contabilidade, Universidade de São Paulo, São Paulo, 1993. 
CLAXTON, Charles S.; MURRELL, Patrícia H. Learning styles: inplications for improving practices. ASHE-ERIC Higher Education Report, n. 4, 1987.

CAVAlHEIRO, M. T. P.; GUIMARÃES, A. L. Formação para o SUS e os Desafios da Integração Ensino Serviço. Caderno FNEPAS; Vol.1; Dez, 2011.

CECCIM, R.B.; FERLA, A.A. Educação e Saúde: Ensino e Cidadania como Travessia de Fronteiras. Trabalho, Educação e Saúde; v.6, p.443-56, nov.2008/ fev.2009.

CECILIO, L. C. O. Modelos tecno-assistenciais em saúde: da pirâmide ao círculo, uma possibilidade a ser explorada. Cad. Saúde Pública [online]; vol. 13, n. 3, pp. 469-478, 1997.

DAVIES, S. M.; RUTLEDGE, C. M.; DAVIES, T. C. The Impact of Student Learning Styles on Interviewing Skills and Academic Performance. Teaching and Learning in Medicine, vol. 9, $\mathrm{n}$. 2, p. 131-135, 1997.

DINIZ, D. D. A Interação no Ensino a Distância sob a Ótica dos Estilos de Aprendizagem. Dissertação (Mestrado em Engenharia de Produção), Escola de Engenharia de São Carlos da Universidade de São Paulo, 2007.

FELDER, R. M.; SOLOMAN, B. A. Index of Learning Style, 1991. Disponível em: <http:// www4.ncsu.edu/unity/lockers/users/f/felder/ public/ILSpage.html>. Acesso em: 29 mar. 2012.

FeUerWerker, L. C. M.; Merhy, E. E. Como temos armado e efetivado nossos estudos, que fundamentalmente investigam políticas e práticas sociais de gestão e de saúde? In: MATTOS, R. A.; BAPTISTA, T. W. F. Caminhos para análise das políticas de saúde, 2011. p. 290-305.

FRANCO, T. B.; MERHY, E.E. Cartografias do Trabalho e Cuidado em Saúde. Revista Tempus Actas de Saúde Coletiva, v.6, n.2, p.151-63,
2012.

GODOY, A. Introdução à Pesquisa Qualitativa e suas Possibilidades. Revista de Administração de Empresas - RAE, v.35, n.2, p.57-63, 1995.

GONÇALO, C. R.; BORGES, M. L. Organizações de Saúde Intensivas em Conhecimento: um estudo no contexto de serviços de alta complexidade. Saúde Soc. São Paulo, v.19, n.2, p.449-461, 2010.

GRANITO, R. A. N. Educação à Distância e Estilos de Aprendizagem: elaboração de um protocolo de qualidade para ambientes virtuais de ensino. Dissertação (Mestrado em Administração), Faculdade de Economia, Administração e Contabilidade, Universidade de São Paulo Ribeirão Preto, 2008.

GRASHA, A. Observations on Relating Teaching Goals to Student Response Styles and Classroom Methods. American Psychologist, v. 27, p 144-147, 1972.

GRISI, C. C. H.; BRITTO, R. T. Estilos de Aprendizagem e o Aprendizado em Comerciais de TV: um estudo exploratório com o método de Kolb. FACEF PESQUISA - v. 7, n. 1, 2004.

HAIR Jr., J. F. Fundamentos de Métodos de Pesquisa em Administração. Porto Alegre: Bookman, 2005.

HAIR Jr., J. F.; ANDERSON, R. E.; TATHAM, R. L.; BLACK, W. C. Análise Multivariada de Dados. 5a Ed. Porto Alegre: Bookman, 2005.

KOLB, D. A. Experiential Learning: experience as the source of learning and development. New Jersey: Prentice-Hall, 1984.

KOLB, D. A. Gestão e o Processo de Aprendizagem. In: STARKEY, K. Como as organizações aprendem. São Paulo: Futura, 1997.

KOLB, D. A. Management and the Learning Process. California Management Review, v.38, n.3, p.21-31, Spring, 1976. 
KURI, N. P. Tipos de Personalidade e Estilos de Aprendizagem: proposições para o ensino de engenharia. Tese (Doutorado em Engenharia de Produção), Universidade Federal de São Carlos, 2004.

MEDEIROS, C. A. F. Comprometimento Organizacional: um estudo de suas relações com características organizacionais e desempenho nas empresas hoteleiras. Tese (Doutorado em Administração), Programa de Pós-Graduação em Administração, Universidade de São Paulo, São Paulo, 2003.

MEDEIROS, C. A. F; ALBUQUeRQUE, L. G.; MARQUES, G. M.; SIQUEIRA, M. Um Estudo Exploratório dos Múltiplos Componentes do Comprometimento Organizacional. Revista de Administração, v.11, n.1, p.1-22, 2005.

MEDEIROS, C. A. F; ENDERS, W. T. Validação do Modelo de Conceitualização de Três Componentes do Comprometimento Organizacional (Meyer e Allen, 1991). Revista de Administração Contemporânea - RAC, v.2, n.3, p. 67-87, 1998.

MEYER, J. P. ALLEN, N. J. SMITH, C. A. Commitment to Organizations and Occupations: extension and test of a three-component conceptualization. Journal of Applied Psychology, v. 78, n. 4, p. 538551, 1993.

MEYER, J. P.; ALLEN, N. J. A Three-Component Conceptualization of Organization Commitment. Human Resource Management Review, v. 1, n. 1, p. 61-89, 1991.

MEYER, J. P.; HERSCOVITCH, L. Commitment in the Workplace: toward a general model. Human Resource Management Review, v. 11, p. $299-326,2001$.

MOWDAY, R. T.; PORTER, L.W.; STEERS, R. $M$. Employee-organization linkages - the psychology of commitment, absenteeism, and turnover. New York: Academic Press, 1982.

MOWDAY, R. T.; STEERS, R. M.; PORTER, L. W. The Measurement of Organizational
Commitment. Journal of Vocational Behavior, v. 14, p. 224-247, 1982.

LAWLER III, E. E. From Human Resource Management to Organizational Effectiveness. Human Resource Management. Hoboken, Vol. 44, No. 2, 165-169, Summer 2005.

NUNNEY, D. N. Cognitive Style Mapping. Training and Development Journal, vol. 32 (9), p. 50-57, Sep. 1978.

PATTON, M.Q. Qualitative Evaluation and Research Methods, 2nd Ed., Newbury Park, CA, USA: Sage Publications, 1990.

PAWLOWSKY, P. The Treatment of Organizational Learning in Management Science. In: DIERKES, M. ANTAL, A. B.; CHILD, J.; NONAKA, I. (Eds). Handbook of Organizational Learning and Knowledge. Oxford: Oxford University Press, p. 61-88, 2001.

PESTANA, M.H.;GAGEIRO, J.N.Análise deDados para Ciências Sociais: a complementaridade do SPSS. 3a Ed. Lisboa: Silabo, 2003.

PROBST, G. J. B.; BÜCHEL, B. S. T. Learning Organization: the competitive advantage of the future. London: Pretence Hall, 1997.

REIS, L. G.; PATON, C.; NOGUEIRA, D. R. Estilos de aprendizagem: uma análise dos alunos do curso de ciências contábeis pelo método Kolb. Revista Enfoque: Reflexão Contábil UEM Paraná v. 31 n. 1 p. 53-66 janeiro / abril 2012.

RICHARDSON, R. J. Pesquisa Social: métodos e técnicas. 3ạ Ed. São Paulo: Atlas, 2011.

SALOMÃO, C. S. Mulheres Empreendedoras em Pequenas Empresas: análise dos estilos de aprendizagem e dos estilos de liderança. Dissertação (Mestrado em Engenharia de Produção), Escola de Engenharia de São Carlos, Universidade de São Paulo, São Paulo, 2011.

SAMPIERI, R. H.; COLLADO, C. F.; LUCIO, P. B. Metodologia da Pesquisa. São Paulo: McGrawHill, 2006. 
SANTOS, D. L. Análise da relação entre o comprometimento e a aprendizagem organizacional: um estudo em uma organização hospitalar. Santa Maria, RS, 2009. Dissertação (Mestrado em Administração) pela Universidade Federal de Santa Maria, 2009.

SILVA, D. M.; NETO, J. D. O. O Impacto dos Estilos de Aprendizagem no Ensino em Contabilidade. Revista Contabilidade Vista \& Revista, v. 21, n. 4, p. 123-156, out./dez. 2010.

TERRA, J. C. C. Gestão do Conhecimento: o grande desafio empresarial: uma abordagem baseada no aprendizado e na criatividade. São Paulo: Negócio Editora, 2001.

UNIVERSIDADE FEDERAL DE SANTA MARIA. Centro de Ciências da Saúde, Hospital Universitário de Santa Maria, 4a Coordenadoria Regional de Saúde - RS, Secretaria de Município da Saúde de Santa Maria. Projeto: Residência multiprofissional integrada em gestão e atenção hospitalar no sistema público de saúde. Santa Maria; 2010.

WIENER, Y. Commitment in Organizations: a normative view. Academy of Management Review, v. 7(3), p. 418-428, 1982.

WITKIN, H. A.; MOORE, C. A.; GOODENOUGH, D. R.; COX, P. W. Field Dependent and Field Independent Cognitive Styles and Their Educational Implications. Review of Educational Research, v. 47, p. 1- 64, 1977.

YIN, R. K. Estudo de Caso: planejamento e métodos. 4a Ed. Porto Alegre: Bookman, 2010. 
